\title{
Historia de la arquitectura y el urbanismo en bicicleta. Ibagué, Colombia 2015-2017
}

\begin{abstract}
Resumen
Los modelos contemporáneos de educación implican la conjunción de varios factores como el aprendizaje simbólico, los hábitos saludables, la disminución de los niveles de contaminación y la transformación de las instituciones educativas para aproximar la academia a las realidades, de modo que se concreten en la transformación de la sociedad y las ciudades. Para este propósito, se presenta el caso de las clases de historia de la arquitectura y el urbanismo en bicicleta, desarrolladas en una ciudad colombiana intermedia, con las limitantes de ausencia de infraestructura y cultura ciclista. Se presentan reflexiones sobre la implementación de esta metodología de aprendizaje que permite motivación, recuerdo, impacto urbano y activismo sobre las políticas públicas, con lo cual se generan espacios replicables en otras ciudades con estas mismas condiciones.
\end{abstract}

Palabras clave: Bicieducación.

Bicinfraestructura. Historia del Urbanismo.

Historia de la Arquitectura. Pedagogía.

\author{
Andrés Ernesto Francel Delgado \\ Doctor en Historia por la Universidad Nacional de \\ Tres de Febrero (UNTREF, Argentina). \\ Posdoctorante en Estudios de Arte y Arquitectura \\ (CIPOST-FACES), de la Universidad Central de \\ Venezuela (UCV). Profesor de la Universidad del \\ Tolima (UT-Colombia). Director del Grupo de \\ Investigación E-Arc \\ (Estudios de Arquitectura y Ciudad). \\ Ibagué - COLOMBIA \\ aefranceld@ut.edu.co \\ orcid.org/0000-0002-3249-3191
}

\section{Para citar este artículo:}

FRANCEL, Andrés. Historia de la arquitectura y el urbanismo en bicicleta. Ibagué, Colombia 20152017. Tempo e Argumento, Florianópolis, v. 11, n. 26, p. 157 - 182, jan./abr. 2019. 


\title{
History of architecture and urbanism by bike. Ibague, Colombia 2015-2017
}

\begin{abstract}
Contemporary education models involve a combination of several factors such as the symbolic learning, healthy habits, lower pollution levels and transformation of educational institutions to bring academia to the realities, so that they materialize in the transformation of society and cities. For this purpose, the case of the history of architecture and urban planning by bicycle, developed in an intermediate Colombian city, with the limitations of lack of infrastructure and cycling culture, is presented. Reflections on the implementation of this learning methodology that allows motivation, memory, urban impact and activism on public policies are presented, with which replicable spaces are generated in other cities with these same conditions.
\end{abstract}

Keywords: Bikeability; bike infrastructure; History of urbanism; History of architecture; pedagogy.

\section{Introducción}

Ibagué es una ciudad de 500.000 habitantes, capital del Departamento del Tolima, situada en el centro de Colombia (FRANCEL, 2015). Tiene una elevación de 1.285 metros sobre el nivel del mar y una temperatura media de $24^{\circ} \mathrm{C}$, con una oscilación de $30^{\circ} \mathrm{C}$ a mediodía y $18^{\circ} \mathrm{C}$ en las noches. Su topografía es quebrada hacia el occidente, norte y sur. Las zonas planas se ubican hacia el oriente, que es la menos urbanizada actualmente. Su topografía corresponde con la elevación del Nevado del Tolima en la cordillera central, al occidente, y la depresión del río Magdalena al oriente. Así, se puede observar una altura de 1.285 mosl en la zona antigua (occidente), 1.040mosl hacia el perímetro urbano, y menos de 90omosl en la zona de expansión (oriente) [Figura 1]. 


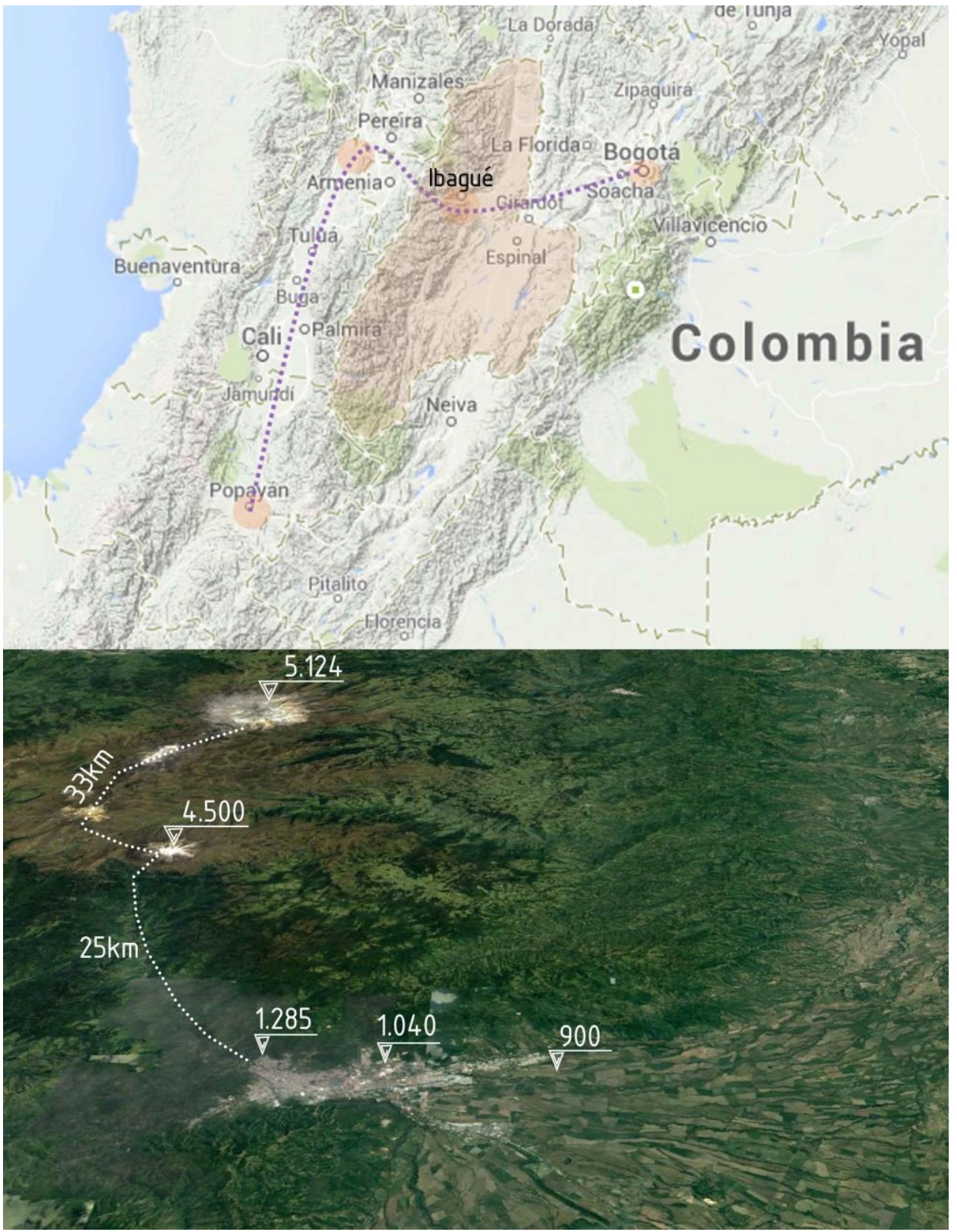

Figura 1. Localización de Ibagué y la topografía general.

Elaboración propia (2018) sobre imágenes de Google Earth.

No es común el uso de la bicicleta como medio de transporte en Ibagué. Existen diversos argumentos topográficos, climáticos, de seguridad e infraestructurales, aunque no hay estudios específicos ni comparativos al respecto (EDITORIAL ELOLFATO.COM, 2016). Recientemente se ha promovido el uso de las bicicletas gratuitas compartidas mediante iniciativas privadas, como el Centro Comercial La Estación (EDITORIAL ELOLFATO.COM, 2016), y públicas como las dispuestas por la Alcaldía de la ciudad (SECRETARÍA DE TRÁNSITO, 2017). 
Estas estrategias se sintonizan con dinámicas de mayor cobertura como la realización del Foro Nacional de la bicicleta en Ibagué (HERRERA VALDÉS, 2016), que tuvo como sede al edificio del panóptico -uno de los bienes de interés cultural más importantes de la ciudad (CIFUENTES SEGOVIA, 2015)- y la participación oficial de la Alcaldía de Ibagué en el Foro Mundial de la bicicleta en Perú (SECRETARíA DE PLANEACIÓN, 2018). Sin embargo, es claro que en la cotidianidad urbana, el ciclista se expone a múltiples peligros por la ausencia de un sistema de ciclismo urbano (CORREA C., 2017; CAÑAS, 2017). En este sentido, se observan las contradicciones entre la creación de políticas y la incapacidad administrativa para implementarlas, como una constante histórica en los procesos de planeamiento local (FRANCEL, 2017).

La academia es el laboratorio en el cual interactúan estas dificultades topográficas, infraestructurales, climáticas y políticas, por lo cual se implementó una metodología experimental de aprendizaje denominada biciclase, o clases de historia de la arquitectura y el urbanismo en bicicleta a partir de 2015. Su propósito esencial fue la reunión del conocimiento académico con la experiencia directa sobre la ciudad, el incentivo de conductas saludables, y el uso de medios de transporte no contaminantes. Las clases en bicicleta se realizan cuatro veces al año y corresponden a sesiones oficiales del ciclo lectivo del programa de arquitectura de la Universidad del Tolima.

El proceso experimental consistió en la realización de la actividad para determinar su grado de asimilación, convocatoria y efectividad en varios campos. De acuerdo con su desarrollo, se tomaron datos sobre las potencialidades y debilidades encontradas. Es fundamental resaltar que al iniciar las biciclases, el profesor guía no tenía experiencia ciclista, por lo cual se desarrolló un proceso de construcción compartida de conocimiento experiencial. Así que en los apartados temáticos se encontrarán las etapas y las reflexiones derivadas del ejercicio académico y ciudadano que se ha expandido hacia ámbitos nacionales.

Los retos que implica esta decisión académica atienden algunas preguntas sobre ¿cómo realizar actividades académicas en bicicleta en una ciudad que carece de infraestructura para su desarrollo? ¿Cómo reforzar el conocimiento sobre el urbanismo y la arquitectura universal a partir de un enfoque en la historia local? ¿Cómo evaluar el 
En primera instancia, esta propuesta busca la generación de posibilidades de enseñanza para garantizar el cumplimiento de algunas expectativas generacionales, lo que significa que se puedan brindar las mejores condiciones para la enseñanza y el aprendizaje en un momento especifico de la historia (FRANCEL, 2015). En consecuencia, actualmente varios paradigmas se entrecruzan, y de su conjunción se generan propuestas para un tipo de educación que vincule la tecnología, la salud, la eficiencia urbana y la relación física y simbólica del hombre con su entorno (RINCÓN ALONSO, 2017).

\section{Materiales y métodos}

Al hablar de una metodología para el desarrollo de las biciclases, se hace referencia a una reflexión teleológica del suceso, que significa la búsqueda de las causas o los procesos guía, más que las convenciones disciplinares o profesionales (INAM, 2002). De acuerdo con los componentes teóricos, se observa que el fundamento de las clases en bicicleta es la innovación incremental, consistente en el rediseño y refuerzo de las características actuales de un proceso, producto o empresa, para lo cual es necesaria una constante experimentación y mejora continua (JIMÉNEZ JIMÉNEZ e SANZ VALLE, 2006). Así, existían algunas actividades que se realizaban simultáneas, pero aisladas, como las periurbias, o caminatas pedagógicas (RODRíGUEZ SÁNCHEZ, 2015), los bicipaseos o tures, coordinados por asociaciones de ciclismo que, generalmente, acometen rutas en las zonas rurales, debido a los retos físicos que implican y al mismo alejamiento del entorno urbano (LUQUE VALLE, BAENA-EXTREMERA e GRANERO-GALLEGOS, 2011), junto con las perspectivas de proyección social del conocimiento desde la universidad con un enfoque en los valores cívicos (BONHAM e KOTH, 2010).

Para la realización de esta innovación incremental se implementó la técnica sinéctica de la analogía personal, consistente en la pregunta: ¿Qué haría yo si...? (GORDON, 1961). De este modo, los bicitoures se trasformaron en clases en las que se puede pedalear y compartir conocimiento sobre la ciudad, mientras se orienta una 
cátedra tradicional. La generación final del nombre o título de las biciclases también corresponde con la analogía simbólica para la búsqueda de algún lema o palabra que puede generar un vínculo rápido y constante con el público (HEMSLEY-BROWN e GOONAWARDANA, 2007).

En segunda instancia, se aplicó la observación directa de acuerdo con el comportamiento de los estudiantes en las clases de historia del arte y la arquitectura. Aunque la atención es alta en los estudiantes, su componente teórico implica que algunos de ellos toman siestas durante las sesiones debido al cansancio generado por la carga académica que implica el currículo de arquitectura, a pesar de las adecuadas condiciones de diseño de los salones (GISLASON, 2010).

La observación participativa también muestra el agotamiento de los profesores por las rutinas y por la necesidad propia de generar entornos más contemporáneos de aprendizaje para diseñar prácticas que promuevan el movimiento (DECLERCQ e CRANZ, 2014). Se observa también la integración entre estudiantes de diversos cursos para reconocer el territorio en múltiples dimensiones y facilitar la interacción para el desarrollo de habilidades sociales (ZUBIETA e DELFINO, 2010). Esto implicó que para el diseño de las biciclases se involucraron estudiantes de varios semestres que cursaban historia de la arquitectura I, II y III, además de invitar a ciudadanos que quisieran unirse libremente para facilitar la sensibilidad social sobre del modo en que la arquitectura entra en la vida cotidiana (LIFCHEZ, 1987).

La revisión de la bibliografía determinó la necesidad de muchos cambios en la educación que involucran las posibilidades de manejar la atención de los estudiantes desde la sensibilidad docente (JENNINGS e GREENBERG, 2009), los peligros en la salud por el sedentarismo (WARREN, 2010), y la necesidad del incremento de la práctica de algún tipo de actividad física moderada (WORLD HEALTH ORGANIZATION, 2006), para la cual el ciclismo constituye una importante posibilidad (GÖTSCHI, GARRARD e GILESCORTI, 2016). Se incorporó el concepto de aula-ciudad que interpreta el territorio urbano como un escenario de aprendizaje a partir de recorridos urbanos (VALSAGNA e TEJEDOR, 2017), y la solidaridad como un valor para afianzar la responsabilidad social (ARRATIA 
Finalmente, la conjunción de estos aspectos conduce al diseño de una experiencia con un grado balanceado de esfuerzo físico e intelectual, que permita estimular al estudiante y al docente al conocimiento, involucrarse con la ciudad y vincular a los ciudadanos a las clases universitarias. A partir de estas interacciones, la academia se actualiza en la sociedad, y la comunidad incorpora el conocimiento especializado que elaboran los grupos de investigación de la universidad.

\section{Resultados}

La motivación de los estudiantes ha consistido en la experiencia de algo novedoso en cuanto a la fusión del estatismo de la clase con el movimiento del deporte y la posibilidad social que implica salir a pedalear, además del reconocimiento de la geografía de la ciudad, de las relaciones entre topografía y desarrollo económico y social, de la identificación de épocas, estilos, técnicas proyectuales y constructivas, y de la reflexión sobre la ciudad. Todo ello crea un ambiente perfecto para generar estímulos efectivos entre el cuerpo, la mente y la ciudad para rastrear valores y conceptos arquitectónicos y urbanísticos. Para comprobar estas apreciaciones, se ha observado en los exámenes orales que existe mayor recordación de los temas vistos en las biciclases que sobre los temas vistos en el aula en cuanto a los detalles y al aprendizaje simbólico.

El mejor método para mantener la motivación y evitar el agotamiento físico y mental durante los recorridos consiste en el pedaleo constante con tres paradas de hidratación, más aquellas derivadas de la semaforización. Para ello, es indispensable un equipo de amplificación básico que permita al guía realizar una charla mientras pedalea suavemente. La relación sugerida es de 3 kilómetros por hora, tomando en cuenta una distancia total de 10 kilómetros, con un ascenso de 130 metros a los 5 kilómetros y un descenso equivalente. Los descensos y ascensos más pronunciados generan mayor adrenalina y menor concentración en los aspectos teóricos. Sin embargo, son fundamentales para plantear momentos de la clase en los cuales se permite cierta 
relajación que beneficie un siguiente momento de disposición física y mental para el aprendizaje [Figura 2].

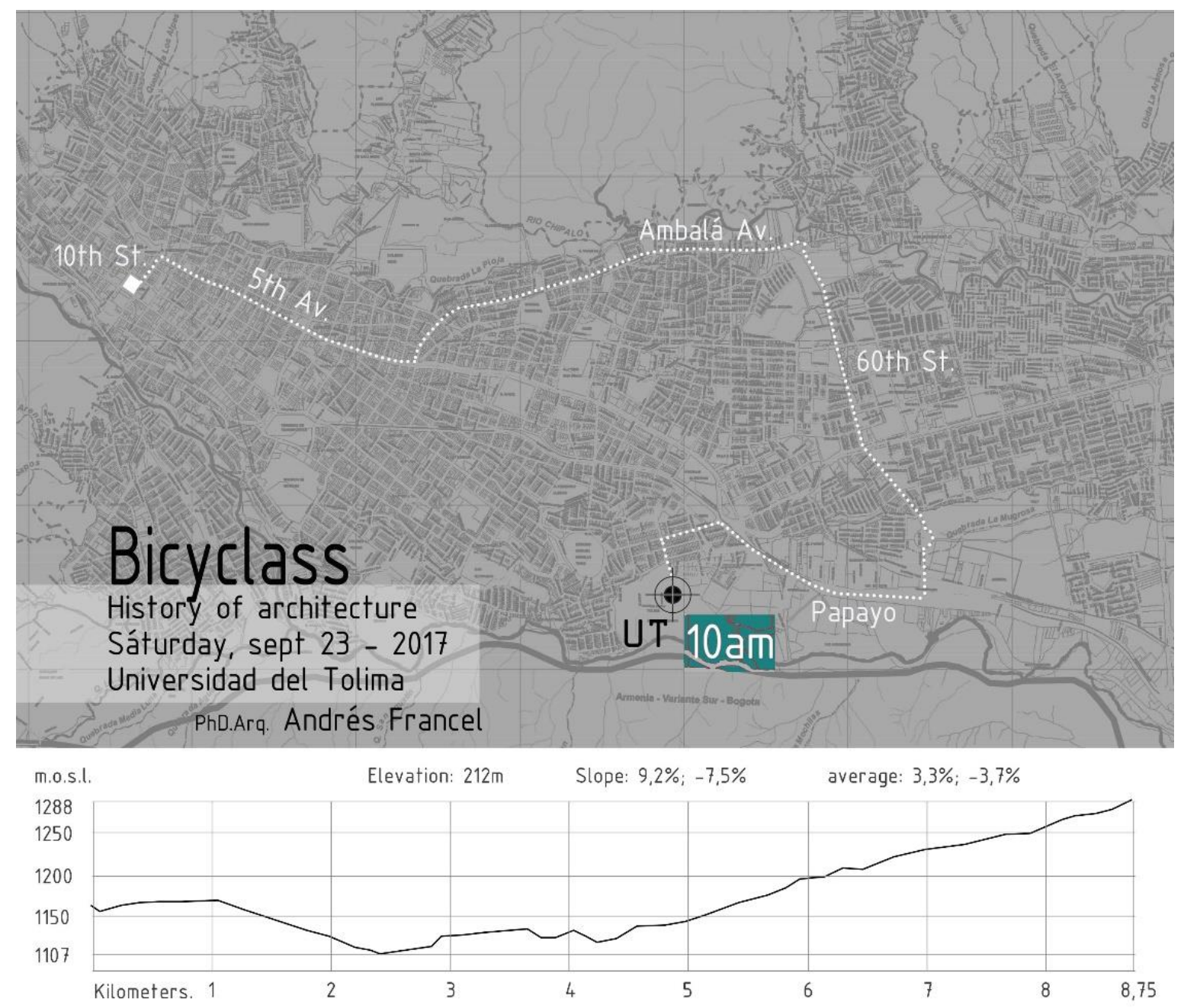

Figura 2. Ruta 2. Elaboración propia (2017) a partir de datos del Google Earth.

La duración recomendada para las biciclases es de tres horas, entre las 9010 de la mañana y las 12 del mediodía o la 1 de la tarde porque permiten condiciones climáticas moderadas y la visibilidad es plena. Se realizan los sábados porque el tránsito desciende y facilita la reunión a los estudiantes en horario extra académico. El horario permite que descansen la noche anterior adecuadamente y almuercen en sus lugares habituales. La reiteración de biciclases también agota, por la cual la intensidad de dos veces por semestre es adecuada. Debe coordinarse con las entregas de taller de diseño para evitar que acontezcan en una misma semana y agoten al estudiante. En general, el orden 
consiste en que el profesor guía se ubica al inicio del grupo, con el parlante en su espalda y los demás pedalean detrás [Figura 3].

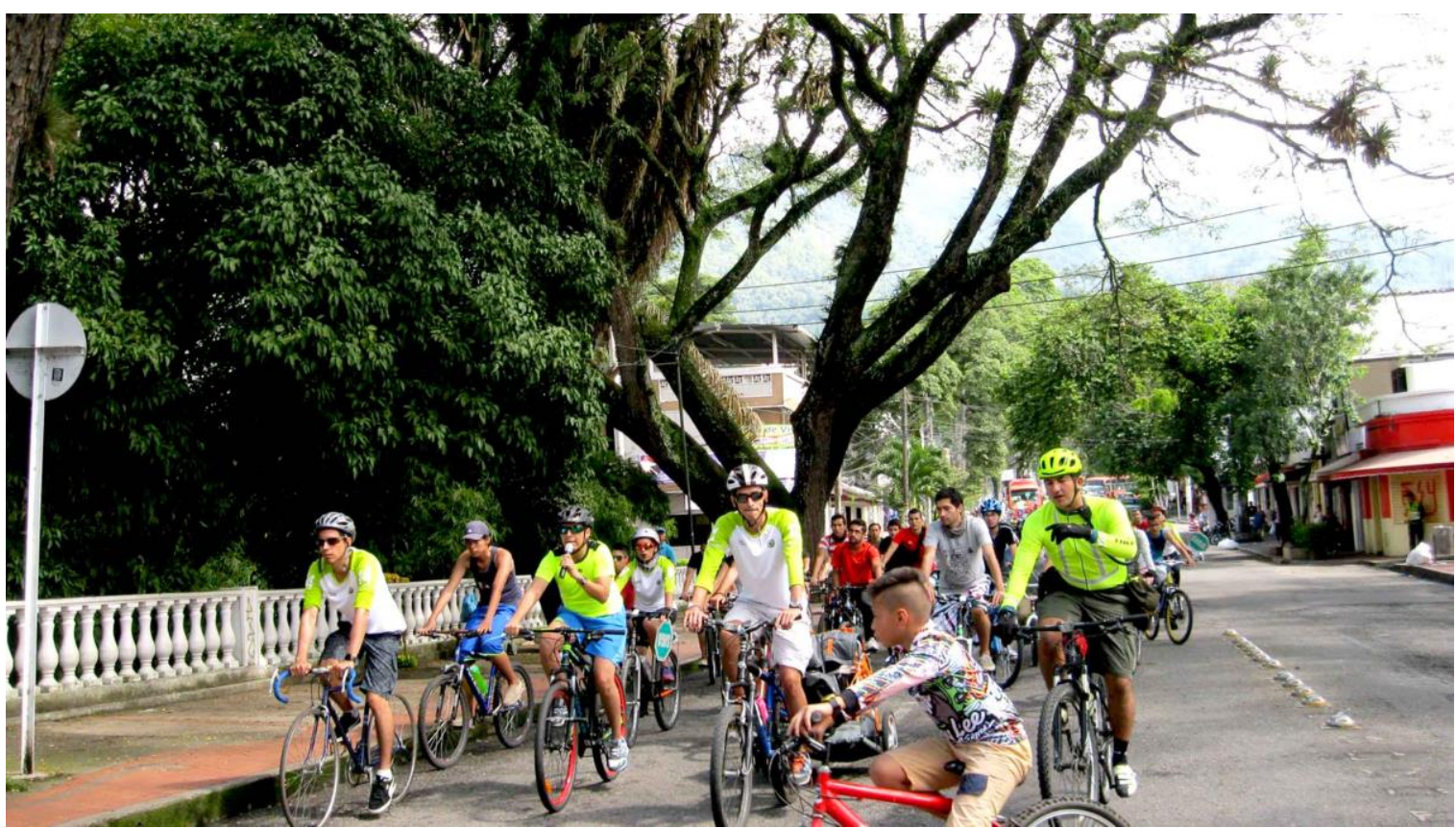

Figura 3. Biciclase con asistencia de 200 personas. Un carril queda libre para el tránsito vehicular. Existe un guía y colaboración de la policía. Se vinculan ciudadanos junto con estudiantes de arquitectura. Fuente: Fundación en tándem (2017).

La iniciativa ha tenido una repercusión social que valida su utilidad, debido a que varias entidades han convocado al docente guía para realizar clases de arquitectura y urbanismo externas a las clases de la Universidad del Tolima. Por ejemplo, La Fundación Entándem convocó una biciclase para el 19 de abril de 2016, entre las 6 y las 9 pm. La Sociedad Colombiana de Arquitectos Regional Tolima convocó una biciclase para el II Encuentro Nacional de Estudiantes de Arquitectura el 11 de marzo de 2017 de 8 a 12 con una asistencia de 500 ciclistas de todo el país (SOCIEDAD COLOMBIANA DE ARQUITECTOS, 2017). La Corporación Unificada Nacional de Educación Superior CUN, convocó una biciclase el 2 de mayo de 2017 entre las 7 y las 10 de la noche, dirigida a los programas de diseño gráficos y textil (ACOSTA, 2017). La Universidad Cooperativa convocó una biciclase en el marco de la semana de la ingeniería civil durante el 10 de mayo de 2017 de las 7 a las 9 pm [Figura 4]. 

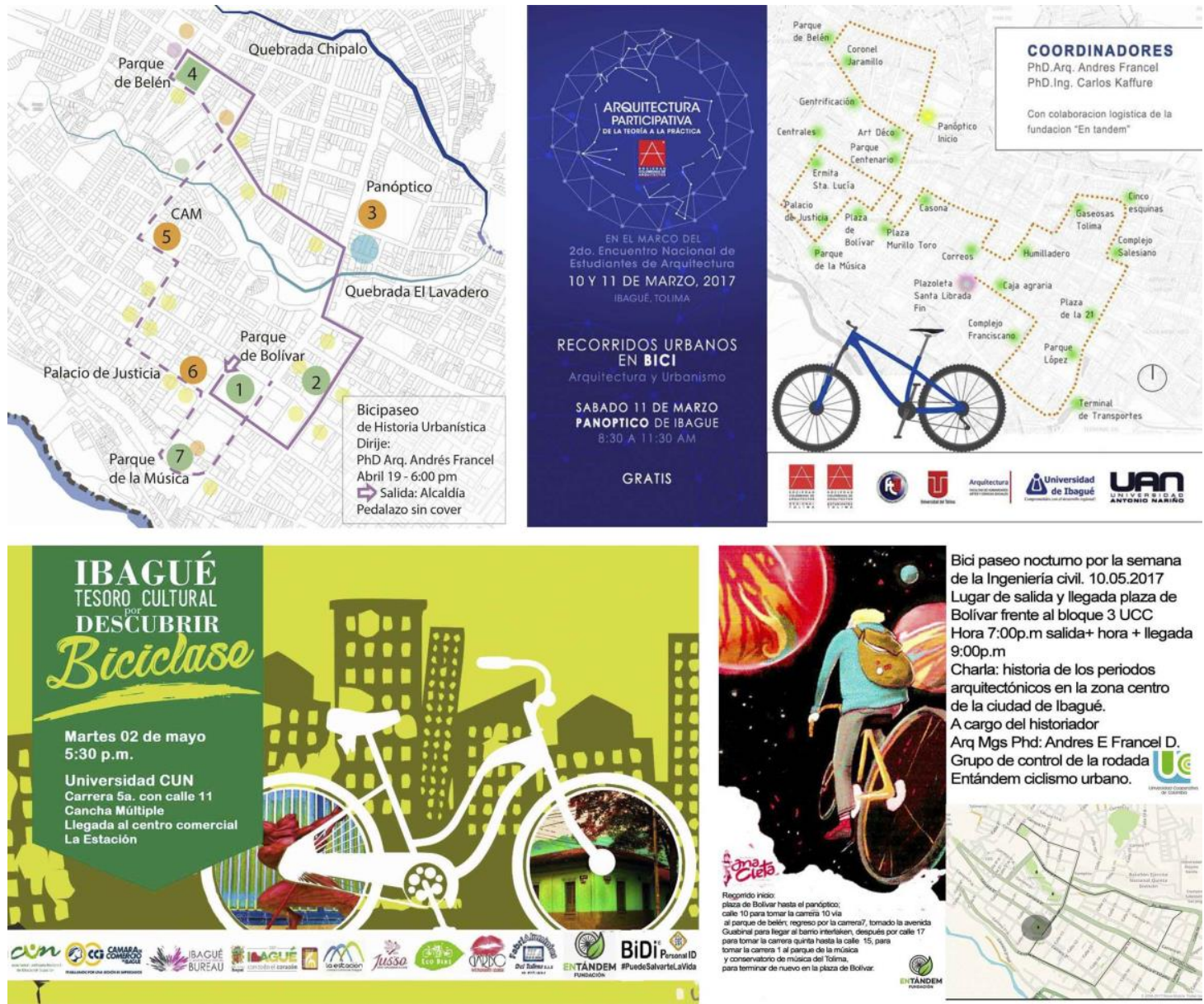

Figura 4. Piezas promocionales de las biciclases externas. Montaje propio (2018) a partir de las imágenes de cada una de las páginas de Facebook de las instituciones.

La vinculación de personas de otras áreas del conocimiento y de otras instituciones muestra también la posibilidad o utilidad como campaña de promoción o publicidad, a la cual es necesaria incorporar el sector turístico que puede generar expansión y rentabilidad futura para el programa. Igualmente, se ha generado un conjunto de piezas promocionales que se pueden ir rastreando las transformaciones en las rutas, las dinámicas utilizadas, frecuencias, y se genera un archivo de investigación sobre las dinámicas de la bicicleta lideradas por el programa de arquitectura. A ellas se suma el perfeccionamiento de la organización mediante las alianzas estratégicas, por ejemplo con la Universidad Cooperativa, mediante docentes catedráticos en las dos universidades; la fundación Entándem, para la coordinación logística relacionada con la pedagogía vial; la policía metropolitana de Ibagué, que ayuda con la preservación de la integridad de los ciudadanos interactuantes; la Sociedad Colombiana de Arquitectos 
Regional Tolima; Asociación Colombiana de Estudiantes de Arquitectura; y, paulatinamente, el gobierno municipal y departamental [Figura 5].

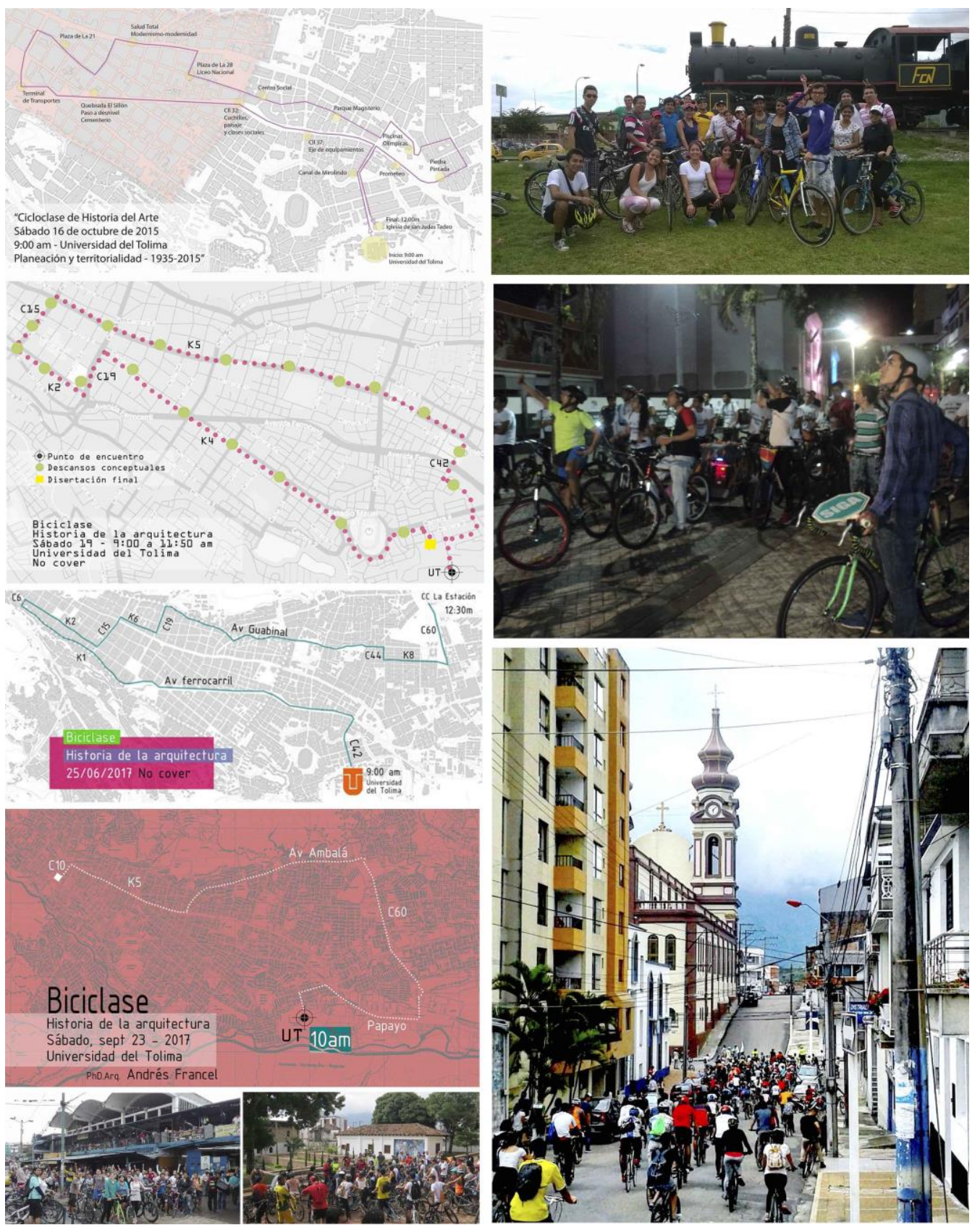

Figura 5. Rutas de las biciclases y fotografías de asistencia. Elaboración propia (2018).

\section{Discusión}

\subsection{El origen}

El primer contacto con la bicicleta y sus actividades grupales fue un bicipaseo organizado por el programa de educación física de la Universidad del Tolima a finales del 
La actividad se basó en los canales de expresión construidos por la mediatización de la cultura moderna (THOMPSON, 2013), como la masa crítica (FURNESS, 2007) del modelo de red, consistente en que los individuos adoptan innovaciones basadas en sus relaciones directas con otros en su sistema social (VALENTE, 1995). De este modo, se pensó en la adaptación de esta actividad al aprendizaje de la historia de la arquitectura, ya que permite la interacción social y la inclusión del componente físico de la ciudad en el sistema social, de un modo más activo que el de las clases tradicionales en un salón.

Algunos meses después, se realizó la primera biciclase, para la cual se convocaron exclusivamente a los estudiantes de los tres semestres en que se cursa de historia del arte y la arquitectura, con el propósito de conformar un grupo interesado en el tema -que fuera numeroso, pero no tan grande que fuera inmanejable. De este modo, se potenciarían las fortalezas de los asistentes y el proyecto tendería a triunfar (OLIVER e MARWELL, 1988). Para el diseño de las rutas es necesario tomar en cuenta la distancia más corta, los descensos, ascensos, la reducción de accidentes por intersecciones, los que se sintetizan en el esfuerzo del ciclista (ISEKI e TINGSTROM, 2013) en relación con el deleite que le produzca su experiencia (WAHLGREN e SCHANTZ, 2012).

La primera sesión de las biciclases permitió observar las características generales de la ciudad, sintetizadas en la ruta diseñada sobre las dos vías con menor pendiente en la ciudad. Una es la avenida del ferrocarril, y la otra la carrera 5. A pesar de ser las más planas, un recorrido de 4 kilómetros entre las calles 12 y 42 presenta un ascenso de 130 metros. En su transcurso no existen bicicarriles, de modo que el ciclista debe invadir las zonas vehiculares y peatonales. Eso significa ser un infractor en donde quiera que se encuentre, fenómeno vinculado a la identificación y medida del transporte enlazado con la exclusión social (LUCAS, 2012) [Figura 6]. 

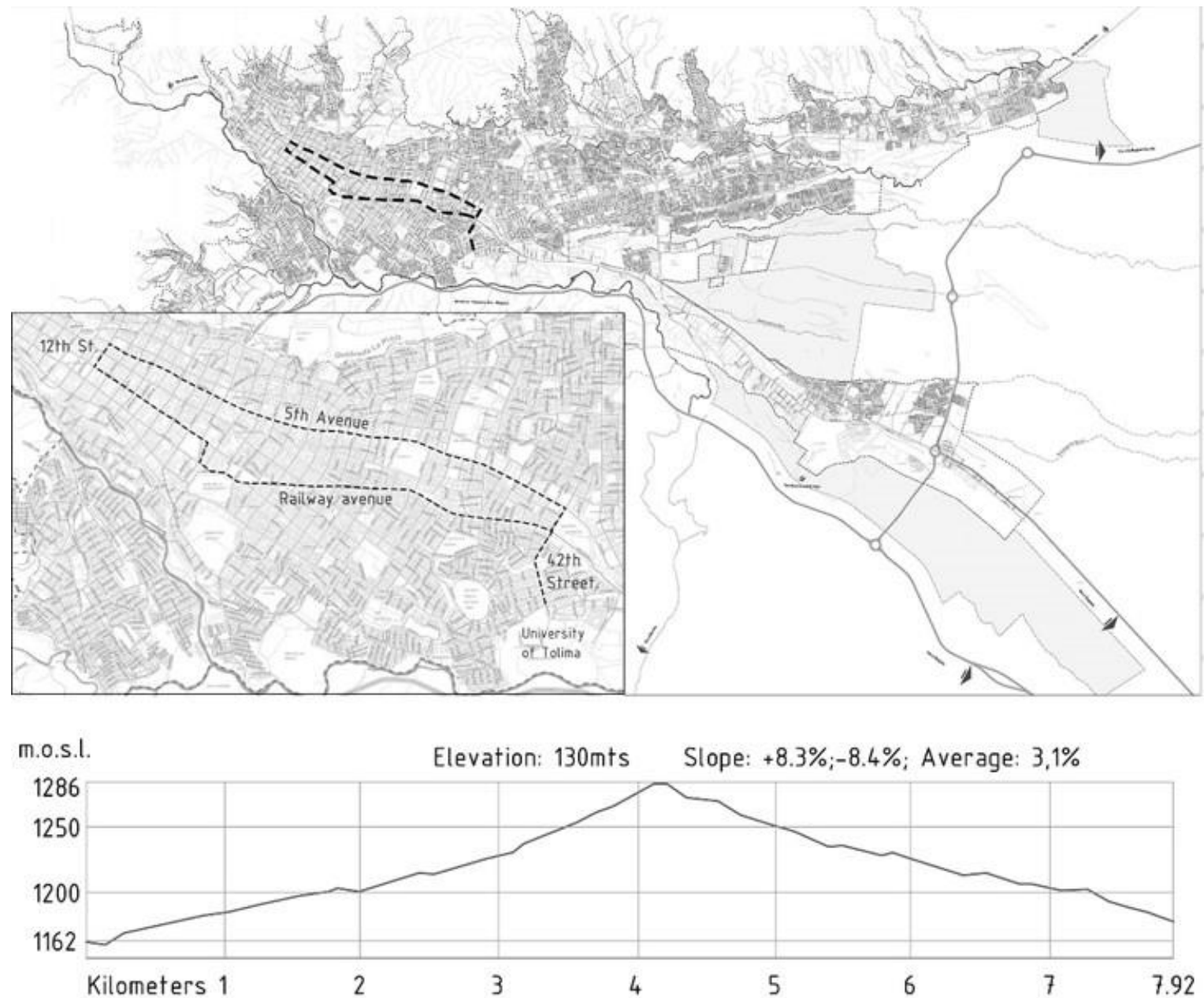

Figura 6. Ruta 1. Elaboración propia (2018) a partir de datos de Google Earth.

\subsection{Revisión de la bibliografía}

El ciclismo urbano es un tema ampliamente abordado, principalmente desde los factores infraestructurales relacionados con la presencia y continuidad de los carriles, los riesgos de los ciclistas (FISHMAN, WASHINGTON e HAWORTH, 2012), la protección del ciclista en las intersecciones, las representaciones porcentuales en el número total de viajes o desplazamientos diarios, la duración comparativa en los trayectos (MARTíN BERMEJO, 2007), la disminución de la polución, la generación de ciudades más amables y la sobreestimación de los vehículos (HOFFMANN, KANDT, et al., 2015).

La exploración de sus posibilidades pedagógicas en el mundo hispanoamericano, prevalece en las asignaturas de educación física (ARRANZ SÁNCHEZ, 2014) y han 
adquirido los títulos de clase en bici en la ciudad de Huesca, consistente en el conocimiento de las características mecánicas, normas y valores desde la asignatura de educación física en España (CASTELLAR OTÍN, PRADAS DE LA FUENTE, et al., 2013). Esta experiencia se deriva del concepto de bikeability, o educación vial para ciclistas en Inglaterra y luego en los Estados Unidos (BERMEJO DEL FRESNO, 2010). Dentro de esta misma lógica, se encuadra Educleta como estrategia comunicativa para profesores de educación física en Santiago de Chile (SANTELICES GÁLVEZ, 2012). En Colombia, figuran las biciclases, implementadas por la institución educativa Rodrigo Lara Bonilla del barrio San Martín de la ciudad de Neiva para enseñar normas de tránsito y convivencia a los estudiantes desde las aulas en Colombia (CORTÉS, 2010).

Con el apoyo de la alcaldía de Bogotá, se generaron las Bici-clases, que nacen en 2012 en el Colegio San Bernardino de la localidad de Bosa en Bogotá, con propósitos definidos, derivados de reflexiones sobre los mecanismos para motivar a los estudiantes por el conocimiento, promoción de la integración entre diversos cursos, reconocer y reconfigurar el territorio para el desarrollo de cuatro dimensiones: corporal, cognitiva, social, intrapersonal y la ruptura de los muros de la escuela mediante en concepto de “ciudad-escuela-ciudad” (LÓPEZ LÓPEZ, MARTíN OCAMPO, et al., 2017).

Biciclase, bici-clase, aula en bici, educleta, cicloclase, bikeability, bicicapacitación y bicieducación. Las variaciones son nominales, pero su concepto esencial es el de aproximarse a un ámbito de relación entre la educación y la ciudad a través de la bicicleta. Sin embargo, los conocimientos transmitidos en estas actividades carecen del enfoque arquitectónico y urbanístico, a pesar de que sea clara la motivación para incorporar la bicicleta como un componente fundamental para el desarrollo (MIRA, CAMASELLE e MARTINEZ, 2003). Principalmente, estas actividades refuerzan las consideraciones sobre la flexibilidad en las direcciones que se pueden tomar en bicicleta, la reducción del caos vehicular y la facilitación de la relación entre comodidad y rapidez en la búsqueda de varios destinos, con un balance entre la peatonalidad y el automovilismo (JENSEN, 2013).

No son comunes en las universidades las opciones de articulación entre la bicicleta y la pedagogía, lo que significa una correspondencia con los enfoques sobre la movilidad, pero no sobre los estudios acerca de la vivencia del lugar desde la arquitectura 
experiencial (RAFFELT, SCHMITT e MEYER, 2013) y las posibilidades del urbanismo contemporáneo en el trabajo colectivo para retomar la ciudad o empoderarse de ella (LYDON e GARCIA, 2015). Estas metodologías convergen en que la arquitectura, el agua y el espacio abierto, constituyen un estimulante impacto independiente de la bicicleta (WAHLGREN e SCHANTZ, 2012).

El enfoque de la historia de la arquitectura y el urbanismo permite abordar conceptos sobre la interpretación del paisaje como rugosidad o testimonio de las historias superpuestas de la sociedad (ZUSMAN, 2002). De este modo, la ciudad es el testimonio de la existencia humana y el medio de validación empírica sobre las teorías de su estudio (SCOTT BROWN, VENTURI e IZENOUR, 2015). También, la aproximación experiencial permite cierta idealización o romantización de los espacios históricos derivados tanto de promoción cultural como de las mismas crisis financieras, a partir de las cuales se fortalecen las construcciones sociales sobre el espacio urbano (DAVIDEL e YUNDA, 2009).

En cuanto al diseño de las rutas para las biciclases, se encontró un principio empírico, consistente en que los "Students are challenged to think critically about what makes a good neighborhood and what contributes to the quality of life of individuals living throughout a large metropolitan area. Students are also encouraged to share their experiences of working and living in the city of Grand Rapids", una experiencia generada desde 2005 y cuyos recorridos recomendados son de 20 millas, o 32 kilómetros (DEGRAAF, 2007). Sin embargo, al realizar el contraste con las características topográficas, climáticas, infraestructurales y culturales de Ibagué, los recorridos recomendados están alrededor de los 10 kilómetros o 6,6 millas, garantizar también velocidades constantes, menores paradas e implica mayor resistencia del guía aunque un pedaleo más suave y sostenido.

\subsection{Architecture bike tours}

Los bicipaseos enfocados en arquitectura son comunes en muchas ciudades del mundo. Con una búsqueda rápida en internet, se pueden encontrar cientos de 
fundaciones alrededor del mundo que los promueven, aunque no son liderados por universidades. En Colombia, Bogotá y Medellín encabezan la promoción de este tipo de actividades, pero son enormes los problemas de seguridad para el ciclista por las deficiencias infraestructurales (MARTÍNEZ ANDRADE, 2017).

Sin embargo, de acuerdo con las condiciones de multitud que representa un bicipaseo urbano (city bike tour), la mayoría de las infraestructuras ciclistas son escasas, ya que con una asistencia mínima de 30 personas es imposible un tránsito lineal por un bicicarril, tomando en cuenta que el pedaleo va acompañado de una charla sobre las construcciones y las características urbanas. Esta característica iguala parcialmente las condiciones para la realización de tures multitudinarios en el primer y tercer mundo, a pesar de las deficiencias infraestructurales, porque las actividades deben ser realizadas en espacios con un ancho mayor a 2,5 metros para que se puedan formar líneas de 304 bicicletas y para que la distancia entre los dos extremos de la longitud permita la difusión adecuada del sonido. Por ejemplo, para un grupo de 30 estudiantes en bici, existirá un ancho de 2,5 metros por una longitud de 15 metros, aproximadamente.

Esta adaptación local de los bicipaseos arquitectónicos (architecture bike tours) presenta reflexiones importantes sobre las condiciones de similitud y disparidad entre las ciudades preparadas para el ciclista y aquellas que carecen de infraestructura para las bicicletas, como el caso de Ibagué (FRANCEL, 2017). Principalmente porque muestra que las actividades de apropiación social masiva de la ciudad no requieren tanto de infraestructura como de reunión de voluntades. Sin embargo, estas actividades espontáneas e inusuales no solucionan los problemas del transporte cotidiano, para los cuales es fundamental la infraestructura.

\subsection{Bici y aprendizaje}

Una de las características más importantes de las clases en bicicleta es la posibilidad de reflexionar sobre el entorno urbano inmediato. Las asignaturas de historia del arte y la arquitectura se conforman por contenidos principalmente internacionales o universales, en atención a la necesidad de fundamentar el repertorio objetual del 
desarrollar criterios propios de análisis que permiten estudiar la incidencia en los factores cognitivos del aprendizaje simbólico como aporte para la enseñanza y para el reconocimiento de las historias locales (FRANCEL, 2017).

Uno de los argumentos pedagógicos más útiles para el ejercicio de las biciclases, se encuentra en las reflexiones de Jerome S. Bruner, quien ha distinguido tres modos básicos mediante los cuales el hombre y la mujer se vuelven a presentar (representar) la realidad. El primero es el modo enactivo, que consiste en la representación de un objeto mediante la reacción inmediata con la realidad. Por ejemplo, al montar una bicicleta, uno se representa la bici. El segundo modo es el icónico, caso para el cual se utilizan imágenes o esquemas para representar el objeto. Por ejemplo, un dibujo puede representar una bici. Finalmente, el modo simbólico representa un objeto mediante un símbolo arbitrario. Por ejemplo, representar una bici mediante la palabra bici (GUILAR, 2009).

Estas observaciones significan la construcción de currículos en espiral, que representan modos de profundizar más y mejor en un determinado corpus de conocimiento en función del entendimiento que corresponda al desarrollo cognitivo del alumno. Por ejemplo, para profundizar más y mejor en el conocimiento de la bicicleta, se realiza primero una acción con ella, que es montar en la bici. Después, se aplican representaciones gráficas como los planos de los recorridos urbanos, los bocetos o ideogramas sobre las obras arquitectónicas estudiadas. Finalmente, los estudiantes deben estar en capacidad de generar definiciones de la bicicleta como instrumento pedagógico y como pretexto para la creación de conocimiento (GUILAR, 2009).

La posibilidad de observar y experimentar la ciudad desde otra óptica que implica factores sensoriales (visuales, táctiles, olfativos, auditivos), conduce a la generación de lecturas de paisaje inhabituales (CÁRDENAS-SOLER e MARTÍNEZ-CHAPARRO, 2015). Estas experiencias implican también redescubrimientos de la ciudad y la generación de conceptos que permiten tanto la valoración arquitectónica y urbanística como reflexiones sensatas sobre el planeamiento urbano (GARCÍA VÁZQUEZ, GONZÁLEZ MARTíNEZ e 
SIERRA HERNÁNDEZ, 2014). La reconfiguración del concepto de aula-ciudad (pista-ciudad, o sus equivalentes) refuerza la interacción entre el arquitecto y el entorno que habita para reflexionar constantemente como peatón, ciclista o conductor, para que le permita ir más allá del medio de transporte y se dirija hacia el sentido del lugar (DARNTON, 2016).

La incorporación o fusión de prácticas físicas a las prácticas académicas tradicionales genera también una conciencia sobre la unión de las dimensiones del cuerpo humano e invita a la búsqueda de opciones que vinculen aspectos físico-académicos que benefician a toda la sociedad. Muchos estudiantes no tienen bicicleta, pero el bykeshare, o préstamo de bicicletas, como política pública y estrategias empresariales particulares han mostrado su utilidad y confirman su impacto y rápido crecimiento alrededor del mundo (FISHMAN, 2016). Finalmente, para la medición numérica de la enseñanza, es claro que la incorporación del factor lúdico y simbólico se verifica en el desarrollo de las evaluaciones en las cuales es notorio el grado de aprendizaje durante las biciclases.

\section{Conclusiones}

Las biciclases son propuestas pedagógicas frente a los valores urbanos y al desarrollo de capacidades e interpretación de la ciudad contemporánea. No solucionan los problemas infraestructurales de las ciudades, pero activan la conexión entre la academia y la sociedad para la integración del conocimiento. Dentro de este marco, revelan que las reinterpretaciones de ciudad pueden desarrollarse en ausencia de infraestructura, mediante la coordinación de horarios, actores sociales e institucionales y el ejercicio del activismo social de carácter pedagógico.

La vinculación de personas e instituciones académicas, culturales y gubernamentales, tanto públicas como privadas, externas a la universidad que originó el concepto de biciclase, es un indicador fundamental sobre el éxito de la propuesta, para la cual no ha existido un plan de medios, sino que su desarrollo ha sido espontáneo, orgánico. En este sentido, crece y se visibiliza en la medida en que la sociedad lo incorpora a sus dinámicas. 
El fenómeno de las biciclases se replica rápidamente en grupos sociales diversos de acuerdo con el concepto esencial de un vehículo sencillo e ideas para compartir mientras se ejercita. Por ello, conduce una resignificación especial de la bicicleta como herramienta pedagógica y de la pedagogía como canal de conexión social. Así, el vehículo y la historia de la arquitectura y el urbanismo logran la construcción de currículos en espiral, mediante los cuales se profundiza en la estructura social del conocimiento de la ciudad.

\section{Referencias}

ACOSTA, Diomedes. La biciclase, una experiencia sobre ruedas, Ibagué, o4 mayo 2017. Disponible: https://soundcloud.com/user-73430629/la-biciclase-una-experiencia-sobre-ruedas. Accesso en: 10 may. 2017

ARRANZ SÁNCHEZ, Beatriz. La bicicleta como recurso educativo en los centros escolares. Valladolid: Facultad de educación y trabajo social, Universidad de Valladolid, 2014. Disponible: http://uvadoc.uva.es/bitstream/10324/7360/1/TFG-G\%20805.pdf. Accesso en: 20 jun. 2016.

ARRATIA FIGUEROA, Alejandrina. Ethics, solidarity and "service learning" in higher education. Acta bioethica, http://dx.doi.org/10.4067/S1726-569X2008000100008 v. 14, n. 1, p. 61-67, 2008. Acceso en: 30 jun. 2016.

BERMEJO DEL FRESNO, Daniel. Bikeability: un nuevo concepto de educación vial en las escuelas. Revista Digital de Educación Física, v. 1, n. 5, p. 6-19, jul./agosto 2010. ISSN 19898304. Disponible: https://dialnet.unirioja.es/servlet/ articulo?codigo=3292120. Accesso en: 15 jun. 2016.

BONHAM, Jennifer; KOTH, Barbara. Universities and the cycling culture. Transportation research part D: transport and environment, v. 15, n. 2, p. 94-102, 2010. Disponible http://www.sciencedirect.com/science/article/pii/S136192090900114X. Accesso en: 05 abr. 2016.

CAÑAS, Angie. Únase esta tarde al paseo en bicicleta para conmemorar a las víctimas del accidente de tránsito. RubenDarioCorrea.Com, Ibagué, 18 noviembre 2017. Disponible: http://rubendariocorrea.com/wpsite/unase-esta-tarde-al-paseo-en-bicicleta-para-conmemorara-las-victimas-de-accidente-de-transito. Accesso en: 15 jan. 2018.

CÁRDENAS-SOLER, Ruth Nayibe; MARTÍNEZ-CHAPARRO, Dennys. El paisaje sonoro, una aproximación teórica desde la semiótica. Revista de Investigación, Desarrollo e Innovación, v. 5, n. 2, p. 129-140, enero/jun. 2015. Disponible:

http://uptc.metarevistas.org/index.php/investigacion_duitama/article/view/3717/3271. Accesso en: 08 sep. 2016. 
CASTELLAR OTÍN, Carlos. et al. Aula en bici: un proyecto longitudinal de intervención docente en Ed. Primaria. Retos. Nuevas tendencias en Educación Física, Deporte y Recreación, n. 23, p. 5-9, 2013. Disponible: http://www.redalyc.org/html/3457/345732289001/. Accesso en: 10 nov. 2016.

CIFUENTES SEGOVIA, Mario Fernando. Retrospectiva constructiva e histórica del Panóptico de Ibagué, Colombia. Apuntes, Bogotá, v. 28, n. 2, p. 16-29, jul./dic. 2015. Disponible: https://doi.org/10.11144/Javeriana.apc28-2.rchp. Acceso en: 05 ago. 2017.

CORREA C., Rubén Darío. 4 ciclistas arrollados en 2 accidentes esta mañana en Ibagué.

RubenDarioCorrea.Com, Ibagué, 11 junio 2017. inicio/judicial. Disponible:

http://rubendariocorrea.com/wpsite/4-ciclistas-arrollados-en-2-accidentes-esta-manana-enibague/. Accesso en: 11 jun. 2017.

CORTÉS, Miller Eduardo. 'La Biciclase', una propuesta para la educación en tránsito.

Ministerio de Educación Nacional de Colombia, 28 abril 2010. Disponible:

https://www.mineducacion.gov.co/observatorio/1722/article-229337.html. Accesso en: 21 abr. 2016.

DARNTON, Phillip. Why do cyclists just talk to themselves? Transport Reviews, v. 36, n. 1, p. 163-166, 2016. Disponible: http://www.tandfonline.com/doi/abs/10.1080/

01441647.2015.1114270?journalCode=ttrv20. Accesso en: 10 oct. 2017.

DAVIDEL, Raluca; YUNDA, Juan. Havana: space through tourism. Gestión y Ambiente, v. 12, n. 1, mayo 2009. Disponible: http://www.redalyc.org/html/1694/169414454010. Accesso en: 12 jul. 2016.

DECLERCQ, Caitlin; CRANZ, Galen. Moving beyond seating-centered learning environments: opportunities and challenges identified in a POE of a campus library. The Journal of Academic Librarianship, v. 40, n. 6, p. 574-584, 2014. Disponible:

http://www.sciencedirect.com/science/article/pii/S0099133314001797. Accesso en: 14 ago. 2016.

DEGRAAF, Don. Liberal arts and place: using walking and biking tours to connect students to place. Strengthening liberal arts education by embracing place and particularity. [s.n.:s.l.], 2007. p. 90-102. Disponible: http://www.calvin.edu/

admin/provost/engagement/teagle/images/CalvinTeagleWhitepaper.pdf\#page=90. Accesso en: 18 ago. 2016.

EDITORIAL ELOLFATO.COM. Concejal de Ibagué resultó herido en accidente de tránsito. Elolfato.com, 12 diciembre 2016. Disponible: http://www.elolfato.com/ presidente-del-concejode-ibague-resulto-herido-en-accidente-de-transito/. Accesso en: 10 jan. 2017.

EDITORIAL ELOLFATO.COM. Inauguran el primer sistema de transporte de bicicletas gratuitas en Ibagué. elolfato.com, Ibagué, 10 agosto 2016. Disponible: 
http://www.elolfato.com/inauguran-el-primer-sistema-de-transporte-de-bicicletas-gratuitasen-ibague. Accesso en: 10 sept. 2016.

FISHMAN, Elliot. Bikeshare: a review of recent literature. Transport Reviews, v. 36, n. 1, p. 92113, 2016. Disponible: http://www.tandfonline.com/doi/abs/10.1080/ 01441647.2015.1033036. Accesso en: 15 jul. 2017.

FISHMAN, Elliot; WASHINGTON, Simon; HAWORTH, Narelle. Understanding the fear of bicycle riding in Australia. Journal of the Australasian College of Road Safety, v. 23, n. 3, p. 19-27, 2012. Disponible: https://eprints.qut.edu.au/53981/. Accesso en: 10 feb. 2017.

FRANCEL, Andrés. Metodología y reflexiones para la reconstrucción de la historia urbana del barrio belén de Ibagué (1921-1925). En: SIMPOSIO COLOMBIANO DE HISTORIA REGIONAL Y LOCAL HISTORELO, 5. 2015a. Anales [...]. Disponible:

https://www.researchgate.net/publication/332353240_METODOLOGIA_Y_REFLEXIONES_PAR A_LA_RECONSTRUCCION_DE_LA_HISTORIA_URBANA_DEL_BARRIO_BELEN_DE_IBAGUE_19 21-1925. Ibagué: Universidad Nacional de Colombia, 2015a. p. 1-13.

FRANCEL, Andrés. Tensiones ideológicas y materializaciones de una ciudad intermedia a comienzos del siglo XX: paradigmas y repercusiones en la ciudad contemporánea. Ibagué, Colombia (1910-1935). En: SEMINARIO INTERNACIONAL DE INVESTIGACIÓN EN URBANISMO,7. Anales [... ] Disponible: https://upcommons.upc.edu/handle/2117/80271. Barcelona, n. 7, junio 2015b. p. 1-18. Disponible: http://upcommons.upc.edu/bitstream/handle/2117/ 80271/72BCN_FrancelAndres.pdf?sequence=1\&isAllowed=y. Accesso en: 05 jun. 2016.

FRANCEL, Andrés. Historia y patrimonio de la periferia interior de Ibagué. Ibagué: Premio de investigación en patrimonio. Alcaldía de Ibagué: Caza de libros editories, 2017a. 126 p. Disponible: https://www.academia.edu/35357626/

Historia_y_patrimonio_de_la_periferia_interior_de_Ibagu\%C3\%A9. Accesso en: 12 dic. 2017.

FRANCEL, Andrés. Kintsugi urbano en la avenida del ferrocarril. Ibagué, Colombia (1921-1970). En: SEMINARIO INTERNACIONAL DE INVESTIGACIÓN EN URBANISMO, 9. Anales [...]. Disponible en: https://upcommons.upc.edu/handle/2117/108348. Bogotá, 2017b. p. 1-18.

FRANCEL, Andrés. La superposición de cartografía histórica como método de análisis morfológico y toma de decisiones urbanísticas: Ibagué, Colombia, 1935-2016. Urbe, Rev. Bras. Gest. Urbana, Curitiba v. 9, n. 2, p. 1-21, 9 mar. 2017c. Disponible en: http://dx.doi.org/10.1590/2175-3369.009.002.a010. Acceso en: 10 mar. 2017.

FURNESS, Zack. Critical Mass, Urban Space and Vélomobility. Mobilities, v. 2, n. 2, p. 299-319, jul./mayo 2007. Disponible: http://www.tandfonline.com/doi/abs/ 10.1080/17450100701381607. Accesso en: 16 abr. 2016. 
FURNESS, Zack. Critical mass rides against car culture. En: ALLHOFF, Fritz. (Series Editor). Cycling: philosophy for everyone: a philosophical tour de force. Hoboken: Wiley-Blackwell, 2010. p. 134-145. Disponible: https://s3.amazonaws.com/academia.edu.documents/36562779/ Furness-Crit_Mass_Rides_Against_Car_Culture.pdf?AWSAccessKeyld=AKIAIWOWYYGZ2Y53 UL3A\&Expires $=1519314560 \&$ Signature $=$ d9mAeoTQ5CVJ10b9gMrTLu7Svz0\%3D\&response content-disposition=inline\%3B\%2ofilename\%3DCr. Accesso en: 16 abr. 2016.

GARCÍA VÁZQUEZ, Carlos; GONZÁLEZ MARTíNEZ, Plácido; SIERRA HERNÁNDEZ, Manuel. Producción de nueva arquitectura en la ciudad de Sevilla y su relación con el Paisaje. En: GARCÍA VÁZQUEZ, Carlos; GONZÁLEZ MARTÍNEZ, Plácido; SIERRA HERNÁNDEZ, Manuel. Guía del paisaje histórico urbano de Sevilla: estudio temático 11. Sevilla: Consejería de Cultura de la Junta de Andalucía, 2014. p. 96. Disponible: https://repositorio.iaph.es/bitstream/11532/162361 /1/ GPHU_ET_11_nueva_arquitectura_y_relaci\%C3\%B3n_con_paisaje_hco_urbano.pdf. Accesso en: 05 may. 2017.

GISLASON, Neil. Architectural design and the learning environment: a framework for school design research. Learning Environments Research, v. 13, n. 2, p. 127-145, jul. 2010. Disponible: https://link.springer.com/article/10.1007/s10984-010-9071-x. Accesso en: 10 feb. 2016.

GONZÁLEZ SOLAS, Javier. The history as a frame: critic of the histories of the design. Area Abierta, v. 25, n. 1, p. 1-17, 2017. Disponible: https://search.proquest.com/docview/208174478/abstract/5707E4344C06473FPQ/1?accountid =41931. Accesso en: 10 oct. 2017.

GORDON, W. J. J. Synectics: The development of creative capacity. New York: Harper \& Brothers, 1961.

GÖTSCHI, Thomas; GARRARD, Jan; GILES-CORTI, Billie. Cycling as a part of daily life: a review of health perspectives. Transport Reviews, United Kingdom, v. 36, n. 1, p. 45-71, 2016. Disponible: http://www.tandfonline.com/doi/abs/10.1080/ 01441647.2015.1057877. Accesso en: 10 jan. 2017.

GUILAR, Moisés Esteban. Las ideas de Bruner: “de la revolución cognitiva” a la "revolución cultural”. Educere, Mérida (Venezuela) v. 13, n. 44, p. 235-241, 2009. Disponible: https://www.redalyc.org/pdf/356/35614571028.pdf. Accesso en: 23 ago. 2016.

HEMSLEY-BROWN, Jane.; GOONAWARDANA, Shivonne. Brand harmonization in the international higher education market. Journal of Business Research, v. 60, n. 9, p. 942-948, 23 marzo 2007. Disponible: https://doi.org/10.1016/j.jbusres.2007.01.019. Acceso en: 25 ago. 2016.

HERRERA VALDÉS, Mauricio. Foro Nacional de la Bicicleta, Ibagué 2016. Revista Arcadia, Bogotá, 25 agosto 2016. Disponible: http://www.revistaarcadia.com/agenda/ articulo/bicicletaforo-nacional-ibague-tolima-colombia-ambiente-cultura-ciclismo/53605. Accesso en: 25 ago. 2016. 
HOFFMANN, Christian. et al. Toward new urban mobility: the case of London and Berlin. London: London School of Economics and the Innovation Centre for Mobility and Societal Change (InnoZ) GmbH, 2015. Disponible: https://files.Isecities.net/files/2015/09/New-UrbanMobility-London-and-Berlin.pdf. Accesso en: 30 ago. 2016

INAM, Aseem. Meaningful urban design: teleological/catalytic/relevant. Journal of urban design, United Kingdom, v. 7, n. 1, p. 35-58, 2002. Disponible: https://www.tandfonline.com/doi/abs/10.1080/13574800220129222. Acceso: 11 may. 2017.

ISEKI, Hiroyuki; TINGSTROM, Matthew. A GIS bikeability/bikeshed analysis incorporating topography, street network and street connectivity. En: WORLD CONFERENCE ON TRANSPORT RESEARCH, 13. Anales electrónicos [...] Rio de Janeiro, 2013. 20 p. Disponible: http://www.wctrs-society.com/wp/wp-content/uploads/abstracts/ rio/selected/3403.pdf. Accesso en: 15 ago. 2016.

JENNINGS, Patricia; GREENBERG, Mark. The prosocial classroom: teacher social and emotional competence in relation to student and classroom outcomes. Review of Educational Research, (local de publicação) v. 79, n. 1, p. 491-525, 1 mar. 2009. Disponible:

https://doi.org/10.3102/0034654308325693. Acceso en: 20 ago. 2016.

JENSEN, Anne. The power of urban mobility: Shaping experiences, emotions and selves on the bike. En: WITZGALL, Susanne; VOGL, Gerlinda; KESSELRING, Sveng. New mobilities regimes in art and social sciences. [S.I.]: Routledge, 2013. p. 273-286.

JIMÉNEZ JIMÉNEZ, Daniel; SANZ VALLE, Raquel. Innovación, aprendizaje organizativo y resultados empresariales: un estudio empírico. Cuadernos de Economía y Dirección de la Empresa, Madrid, España, n. 29, p. 32-55, 2006. Disponible:

http://www.redalyc.org/html/807/80702902/. Accesso en: 18 sept. 2016.

LIFCHEZ, Raymond. Rethinking architecture: design students and physically disabled people. Los Angeles: University of California Press, 1987.

LONDOÑO SÁNCHEZ, Juan Guillermo; CARVAJAL GUZMÁN, Jeimmi. Pedagogies for the historical memory: reflections and considerations for a process of innovation in the classroom. Ciudad Paz-ando, Bogotá, v. 8, n. 1, p. 124-141, jun. 2015. Disponible: https://revistas.udistrital.edu.co/ojs/index.php/cpaz/article/viewFile/ 8261/10594. Accesso en: 08 ago. 2016.

LÓPEZ LÓPEZ, José Arquímedes. et al. Un encuentro pedagógico con el ser, el saber y el territorio: ¡hagamos Bici-clase! En: CRUZ, César Augusto Pulga et al. Sistematización de experiencias de acompañamiento in Situ 2016: infancia, convivencia y paz, ambientes de aprendizaje y saberes tecnomediados. Bogotá: Instituto para la Investigación Educativa y el Desarrollo Pedagógico: IDEP, 2017. p. 203-228. Disponible: http://www.idep.edu.co/sites/default/files/libros/ Experiencias\%20In\%20situ\%20\%20IDEP\%202017\%20WEB.pdf\#page=203. Accesso en: 08 sep. 2017. 
LUCAS, Karen. Transport and social exclusion: where are we now? Transport Policy, United Kingdom, v. 20, p. 105-113, 14 feb. 2012. Disponible: https://doi.org/10.1016/j.tranpol.2012.01.013. Acceso en: 04 abr. 2016.

LUQUE VALLE, Pablo; BAENA-EXTREMERA, Antonio; GRANERO-GALLEGOS, Antonio. Buenas prácticas para un desarrollo sostenible en los eventos deportivos en el medio natural. Interciencia, Caracas, Venezuela, v. 36, n. 7, jul. 2011. Disponible: http://www.redalyc.org/articulo.oa?id=33919424009. Accesso en: 08 jun. 2016.

LYDON, Mike; GARCIA, Anthony. Tactical urbanism. Washington, DC: Island Press, 2015.

MARTÍN BERMEJO, David. Comparación de tiempos de trayectos metro-a pie-bici en la zona urbana de Barcelona. Barcelona: Universitat Politécnica de Catalunya, 2007. Disponible: http://hdl.handle.net/2099.1/3316. Accesso en: 23 jan. 2016.

MARTÍNEZ ANDRADE, Andrés. Las calles son nuestras: apropiación del espacio público a través del uso de la bicicleta en la ciudad de Medellín. 2017. Trabalho de Conclusão de Curso (Graduação em antropología) - Universidad de Antioquia. Facultad de Ciencias Socieales y Humanas, Medellín. 60 p. Disponible:

http://200.24.17.74:8080/jspui/bitstream/fcsh/1099/1/MartinezAndres_2017_CallesNuestrasApr opiacion.pdf. Accesso en: 11 nov. 2017.

GARCÍA MIRA, Ricardo; CAMASELLE, José; MARTINEZ, José. R. Culture, environmental action, and sustainability. [S.I.]: Hogrefe \& Huber Publishing, 2003. 411 p.

OLIVER, Pamela; MARWELL, Gerald. The paradox of group size in collective action: a theory of the critical mass II. American Sociological Review, United States, v. 53, n. 1, p. 1-8, febrero 1988. Disponible: http://www.jstor.org/stable/2095728. Accesso en: 25 may. 2016.

RAFFELT, Ursula; SCHMITT, Bernd; MEYER, Anton. Marketing function and form: how functionalist and experiential architectures affect corporate brand personality. International. Journal of Research in Marketing, Netherlands, v. 30, n. 3, p. 201-210, september 2013. Disponible: https://doi.org/10.1016/j.ijresmar.2013.02.002. Acceso en: 26 feb. 2016.

RINCÓN ALONSO, José David. Pedaleando por Bogotá: la ciudad como espacio de percepción, reconstrucción y reapropiación. Bogotá. 2017. Pasantía de Investigación (Licenciatura en Educación Básica con énfasis en Ciencias Sociales) - Universidad Distrital Francisco José de Caldas. 2017. 69 p. Disponible: http://repository.udistrital.edu.co/bitstream/ 11349/5633/1/ PEDALEANDO\%20POR\%20BOGOT\%C3\%81.pdf. Acceso en: 11 nov. 2017.

RODRÍGUEZ SÁNCHEZ, Carlos Javier. Transigrafías: caminar como práctica artísticopedagógica. URBS: Revista de Estudios Urbanos y Ciencias Sociales, Madrid, v. 5, n. 1, p. 3556, 2015. Disponible: http://www2.ual.es/urbs/index.php/urbs/ article/view/rodriguez_sanchez/245. Accesso en: 20 jun. 2016. 
SANTELICES GÁLVEZ, Andrés Esteban. Educleta: proyecto que fomenta la sensibilización y valorización del uso de la bicicleta con utilización activa de medios de comunicación dirigido a profesores de educación física de NB3 y NB4 de colegios municipales de la Región Metropolitana. 2012. Tesina (Maestría en Comunicación Social con mención en Comunicación y Educación) - Facultad de Comunicaciones, Pontificia Universidad Católica de Chile, Santiago, 2012. Disponible: https://repositorio.uc.cl/bitstream/handle/11534/891/ 588073.pdf?sequence=1. Accesso en: 12 mar. 2016.

SCOTT BROWN, Denise; VENTURI, Robert; IZENOUR, Steven. Aprendiendo de Las Vegas: el simbolismo olvidado de la forma arquitectónica. Barcelona: Editorial Gustavo Gili, 2015.

SECRETARÍA DE PLANEACIÓN. Ibagué participará en Foro Mundial de la bicicleta. Ibagué: Página oficial de la Alcaldía de Ibagué, 05 febrero 2018. Disponible:

http://www.ibague.gov.co/portal/seccion/noticias/index.php?idnt=4630. Accesso en: 07 feb. 2018.

SECRETARÍA DE TRÁNSITO. Inicia prueba piloto de estación de bicicletas públicas compartidas. Ibagué: Pagína oficial de la alcadía de Ibagué, 03 nov. 2017. Disponible en: http://www.ibague.gov.co/portal/seccion/noticias/index.php?idnt=4050. Accesso en: 15 dic. 2017.

SOCIEDAD COLOMBIANA DE ARQUITECTOS. Encuentro Nacional de Estudiantes de Arquitectura, 2. Analaes electrónicos, Bogotá [... ]. 2017. 10 marzo 2017. Disponivel em: http://sociedadcolombianadearquitectos.org/portfolio/2do-encuentro-nacional-deestudiantes-de-arquitectura/. Accesso en: 18 ago. 2017.

THOMPSON, John. Ideology and modern culture: critical social theory in the era of mass communication. Cambridge: John Wiley \& Sons, 2013.

VALENTE, Thomas. Network models of the diffusion of innovations. Cresskill, New Jersey: Hampton Press, 1995. 171 p.

VALSAGNA, Andrea; TEJEDOR, María Mercedes. De la gestión local de riesgos. La experiencia de la Ciudad de Santa Fe. Medio Ambiente y Urbanización, Buenos Aires, Argentina, v. 86, n. 1, p. 43-69, May 2017. Disponible en: http://www.ingentaconnect.com/contentone/iieal/meda/ 2017/00000086/00000001/art00003\#. Accesso en: 18 ago. 2017.

VENTURI, Robert. Complejidad y contradicción en la arquitectura. Barcelona: Editorial Gustavo Gili, 1995.

WAHLGREN, Lina; SCHANTZ, Peter. Exploring bikeability in a metropolitan setting: stimulating and hindering factors in commuting route environments. BMC Public Health, United Kingdom, v. 12, n. 1, p. 168, 2012. Disponible:

https://bmcpublichealth.biomedcentral.com/articles/10.1186/1471-2458-12-168. Accesso en: 25 mar. 2016. 
WARREN, Tatiana. Et al. Sedentary behaviors increase risk of cardiovascular disease mortality in men. Medicine and science in sports and exercise, Washington D.C., v. 42, n. 5, p. 879-885, 2010. Disponible: <https://www.ncbi.nlm.nih.gov/pmc/articles/PMC2857522/. Accesso en: 30 abr. 2016.

WORLD HEALTH ORGANIZATION. Global strategy on diet, physical activity and health: a framework to monitor and evaluate implementation. Geneva: World Health Organization, 2006. Disponible em: http://apps.who.int/iris/bitstream/10665/

43524/1/9789241594547_eng.pdf. Accesso en: 14 sep. 2016.

ZUBIETA, Elena; DELFINO, Gisela. Satisfacción con la vida, bienestar psicológico y bienestar social en estudiantes universitarios de Buenos Aires. Anuario de investigaciones, Buenos Aires, Argentina, n. 17, p. 277-283, 2010. Disponible en:

http://www.scielo.org.ar/scielo.php?pid=S185116862010000100027\&script=sci_arttext\&tlng=e n. Accesso en: 08 mar. 2016.

ZUSMAN, Perla. Milton Santos: su legado teórico y existencial (1926-2001). Documents d'anàlisi geogràfica, Barcelona, España, n. 40, p. 205-222, 2002. Disponible: http://www.raco.cat/index.php/DocumentsAnalisi/article/view/31765/31599. Accesso en: 22 jun. 2016.

Recebido em 01/06/2018 Aprovado em 17/12/2018

Universidade do Estado de Santa Catarina - UDESC

Programa de Pós-Graduação em História - PPGH

Revista Tempo e Argumento Volume 11 - Número 26 - Ano 2019 tempoeargumento@gmail.com 